\title{
VARIABILITY OF SAGITTARIUS A*: FLARES AT 1 MILLIMETER
}

\author{
Jun-Hui Zhao, ${ }^{1}$ K. H. Young, ${ }^{1}$ R. M. Herrnstein, ${ }^{1}$ P. T. P. Ho, ${ }^{1}$ T. Tsutsumi, ${ }^{1}$ \\ K. Y. Lo, ${ }^{2}$ W. M. Goss, ${ }^{3}$ AND G. C. Bower ${ }^{4}$ \\ Received 2002 October 2; accepted 2003 February 4; published 2003 February 20
}

\begin{abstract}
We report the results from recent observations of Sgr $A^{*}$ at short-/submillimeter wavelengths made with the partially finished Submillimeter Array (SMA) on Mauna Kea. A total of 25 epochs of observations were carried out over the past 15 months from 2001 March to 2002 May. Noticeable variations in flux density at $1.3 \mathrm{~mm}$ were observed showing three "flares." The SMA observations suggest that Sgr A* highly increases toward submillimeter wavelengths during a flare, suggesting the presence of a break wavelength in spectral index around $3 \mathrm{~mm}$. A crosscorrelation of the SMA data at $1 \mathrm{~mm}$ with the Very Large Array data at $1 \mathrm{~cm}$ shows a global delay of $t_{\text {delay }}>$ 3 days, suggesting that submillimeter wavelengths tend to peak first. Only marginal day-to-day variations in flux density $(2-3 \sigma)$ have been detected at $1.3 \mathrm{~mm}$. No significant flares on a short timescale $(\sim 1 \mathrm{hr})$ have been observed at $1.3 \mathrm{~mm}$. We also failed to detect significant periodic signals at a level of $5 \%(3 \sigma)$ from Sgr A* in a periodic searching window ranging from 10 minutes to $2.5 \mathrm{hr}$. The flares observed at the wavelengths between short-centimeter and submillimeter might be a result of collective mass ejections associated with X-ray flares that originate from the inner region of the accretion disk near the supermassive black hole.
\end{abstract}

Subject headings: accretion, accretion disks — black hole physics — galaxies: active — Galaxy: center — radio continuum: galaxies

\section{INTRODUCTION}

Sgr A*, a compact radio source, is believed to be associated with the supermassive black hole at the Galactic center (Eckart et al. 2002; Ghez et al. 2000). The inferred bolometric luminosity $\left(L \sim 10^{-8.5} L_{\mathrm{Edd}}\right)$ is far below the Eddington luminosity for the black hole mass of $\sim 2.6 \times 10^{6} M_{\odot}$. Sgr A* represents an extremely dim galactic nucleus. The low luminosity of Sgr A* is explained by the low efficiency radiative advection-dominated accretion flow (ADAF) model (Narayan et al. 1998). However, the ADAF alone cannot explain the detailed activities, such as the radio outbursts and the X-ray flares. The recent work by Yuan, Markoff, \& Falcke (2002a) provides an alternative model to link a jet outflow (Falcke, Mannheim, \& Biermann 1993) with the ADAF. This new model appears to be able to fit reasonably well the overall spectrum of Sgr A* from radio, submillimeter, IR to X-ray.

The apparent structure at radio wavelengths longer than $3 \mathrm{~mm}$ appears to be mainly dominated by the scattering effects due to the interstellar medium (ISM). At $3 \mathrm{~mm}$, the scattering effects are finally small enough that the intrinsic source size is estimated to be less than 0.27 mas (Doeleman et al. 2001), or about 40 times the Schwarzschild radius $\left(R_{\mathrm{Sc}}\right.$ hereafter). A promising way to explore this extremely compact source is to monitor the variations of the emitted flux density from radio to X-ray.

The variations in the radio flux density of Sgr A* have been known for two decades (Brown \& Lo 1982). The nature of the radio variability has not been well understood. At the long wavelengths, the flux density of Sgr A* might be modulated by the scintillation due to the turbulence in the ISM (Zhao et al. 1989).

\footnotetext{
${ }^{1}$ Harvard-Smithsonian Center for Astrophysics, 60 Garden Street, MS 78 Cambridge, MA 02138; jzhao@cfa.harvard.edu, kyoung@cfa.harvard.edu, rmcgary@cfa.harvard.edu,pho@cfa.harvard.edu,ttsutsumi@cfa.harvard.edu.

2 Academia Sinica, Institute of Astronomy and Astrophysics, P.O. Box 187, Nankang, Taipei 115, Taiwan; flo@nrao.edu.

${ }^{3}$ National Radio Astronomy Observatory, Array Operations Center, P.O. Box O, Socorro, NM 87801; mgoss@aoc.nrao.edu.

${ }^{4}$ Department of Astronomy, University of California at Berkeley, 601 Campbell Hall, Berkeley, CA 94720; gbower@astron.berkeley.edu.
}

The radio light curves observed with the Very Large Array (VLA) at wavelengths from 20 to $1.3 \mathrm{~cm}$ during the period of 1990-1993 suggest that the fractional amplitude variations increased toward short wavelengths and that the rate of radio flares appeared to be about three per year (Zhao et al. 1992; Zhao \& Goss 1993). The typical timescale of these radio flares is about a month. The observed large amplitude variations in flux densities at $3 \mathrm{~mm}$ (Wright \& Backer 1993; Tsuboi, Miyazaki, \& Tsutsumi 1999) are consistent with the wavelength dependence of the variability as observed at centimeter wavelengths.

The presence of a 106 day cycle in the radio variability of Sgr A* was suggested from an analysis of data observed with the VLA in the period of 1977-1999 (Zhao, Bower, \& Goss 2001). The periodic oscillation at a period around 100 days appears to persist in the densely sampled light curves obtained with the VLA at 2, 1.3, and $0.7 \mathrm{~cm}$ over the past $2 \mathrm{yr}$ (J.-H. Zhao et al. 2003, in preparation). The period of the fluctuation cycle appears to increase to $\sim 130$ days (Bower et al. 2002a; J.-H. Zhao et al. 2003, in preparation). In addition, a longer periodic fluctuation feature with a period 2.4-2.5 times the short one has also been seen (J.-H. Zhao et al. 2003, in preparation). Similar periodic fluctuation in flux density was also observed in the Green Bank Interferometer monitoring data (Falcke 1999).

Observations of $\mathrm{Sgr} \mathrm{A}^{*}$ at submillimeter wavelengths can penetrate into the deep region of this intriguing source. In this Letter, we report results obtained from a monitoring program at $1 \mathrm{~mm}$, with the partially finished Submillimeter Array (SMA; Moran 1998).

\section{OBSERVATIONS AND DATA REDUCTION}

Observations of Sgr A* at 1.3 and $0.87 \mathrm{~mm}$ were made using the partially completed SMA with three or four antennas and baselines ranging from 7 to $55 \mathrm{k} \lambda$ at $1.3 \mathrm{~mm}$. A total of 24 epochs of observations at $1.3 \mathrm{~mm}$ were carried out in reasonably good weather conditions (the sky opacity $\tau_{\text {zenith }}<0.3$ at $1.3 \mathrm{~mm}$ ). One observation was made at $0.87 \mathrm{~mm}$ on 2001 March 22 with $\tau_{\text {zenith }} \sim 0.5$ at $0.87 \mathrm{~mm}$. The observations were carried out with a total bandwidth of $328 \mathrm{MHz}$ for each sideband. A typical 


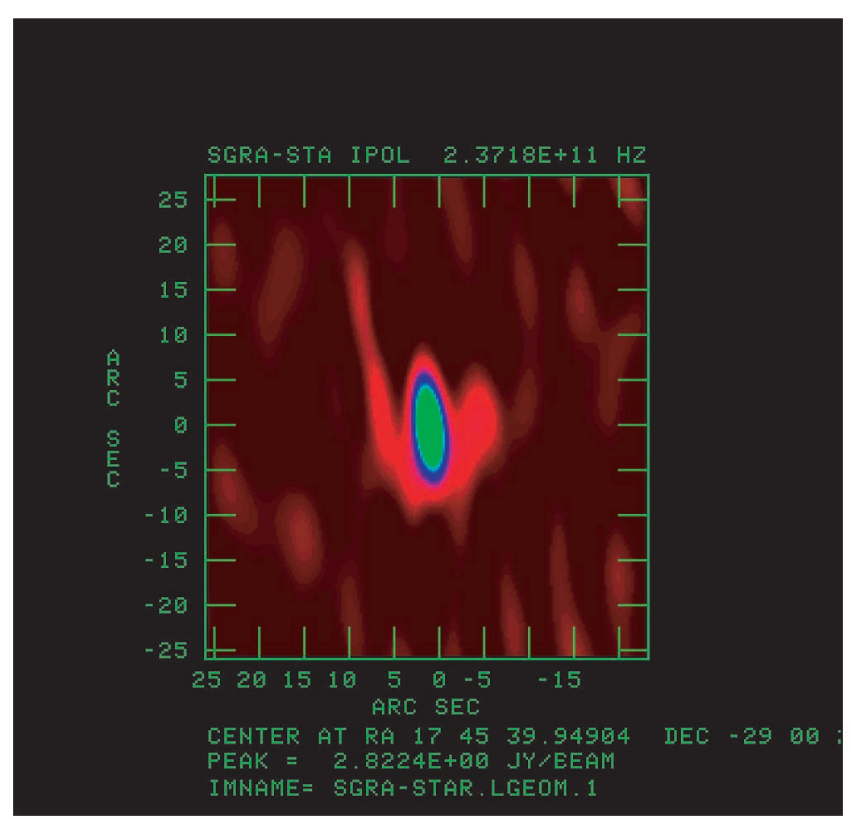

FIG. 1.-Pseudocolor image of Sgr A* (blue) and its vicinity (red) made from $3.5 \mathrm{hr}$ observations using the four elements of the partially finished SMA on 2002 May 23. The total integration time on the source is about $1.5 \mathrm{hr}$. The rms noise is $\sim 30 \mathrm{mJy}^{\text {beam }}{ }^{-1}$. The FWHM beam is $7^{\prime \prime} .4 \times 2.3$ (P.A. $=7^{\circ}$ ). The data reduction was done in the AIPS environment following a procedure described in a SMA technical memorandum (Zhao 2002).

system temperature is $200-300 \mathrm{~K}$ at $1.3 \mathrm{~mm}$. A typical rms noise of $20 \mathrm{mJy}$ was achieved from an observation with four antennas for a typical on-source integration time of $2 \mathrm{hr}$. In each epoch of observation, we interleaved Sgr A* with Sgr B2(N), a compact $\left(<5^{\prime \prime}\right.$ in size) nearby $\left(\sim 1^{\circ}\right) \mathrm{H}$ II region, and two nearby quasistellar objects (QSOs), OV $236\left(\sim 22^{\circ}\right)$ and NRAO $530\left(\sim 16^{\circ}\right)$. The flux density scale in each observation was determined by observing a compact planet (Neptune $[<2$ ".5 in diameter] and Uranus $\left.\left[<4^{\prime \prime}\right]\right)$. Sgr B2(N) ( 50 Jy at $\left.1.3 \mathrm{~mm}\right)$ was used to monitor the stability of the telescope during the observations, for example, the effect due to the possible telescope pointing drift.

Further calibration was done by observing the two QSOs, OV 236 and NRAO 530. We fitted the secular variations of each calibrator with polynomials. Any correlated offsets in flux density from the secular variations of the two QSOs are considered as systematic offsets, such as telescope pointing error. The residual gain correction determined from the offsets are applied to the $\mathrm{Sgr} \mathrm{A}^{*}$ data in order to minimize the systematic errors. The final uncertainty of $\sim 10 \%$ in the flux density calibration is assessed by calculating the standard deviations of the residual offsets from the calibrators. However, the variation of a possible linear polarization as a function of parallactic angle was not corrected. A fractional linear polarization of $\sim 7 \%$ from Sgr A* has been detected (Bower et al. 2002b). The error due to this effect is less significant and is embedded in the final uncertainty.

In addition, $\mathrm{Sgr} \mathrm{A}^{*}$ is embedded in the complex, extended source Sgr A West. Figure 1 shows the image of Sgr A* and its vicinity observed with the SMA at $1.3 \mathrm{~mm}$ with a beam of $7 " .4 \times 2$.".3 (position angle [P.A.] $=7^{\circ}$ ). At this wavelength and this angular resolution, $\operatorname{Sgr} \mathrm{A}^{*}$ is about 10 times brighter than the surrounding components. By examining the visibilities as a function of baseline lengths, we find that, for baselines $\sim 20 \mathrm{k} \lambda$ or longer, Sgr A* is the dominant source, and the confusing flux density at $1 \mathrm{~mm}$ from the surrounding free-free and dust emission is less than $0.3 \mathrm{Jy}$. The flux density measurements were made

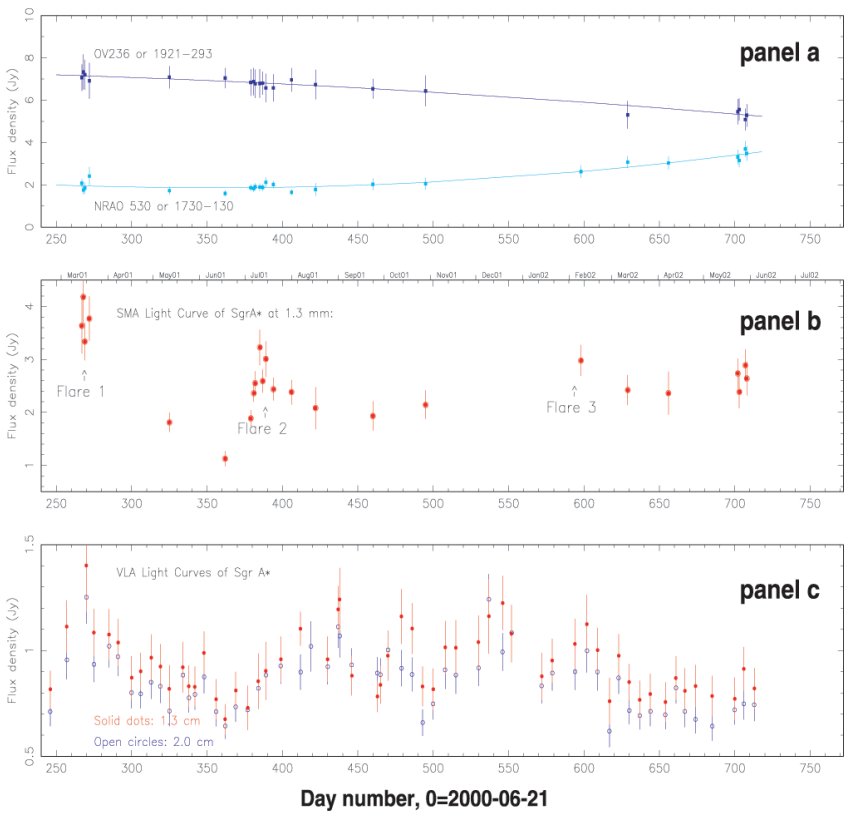

FIG. 2.-SMA light curves at $1.3 \mathrm{~mm}$ observed in the period between 2001 March and 2002 May for the calibrators, OV 236 and NRAO 530 (a) and Sgr A* $(b)$. The solid curves in $(a)$ are the quadratic fits to the secular variations of the flux density of OV 236 and NRAO 530. The secular trends of the flux density from NRAO 530 and OV 236 are the same as those observed at $3 \mathrm{~mm}$ at other observatories (e.g., M. Yun 2002, private communication). (c) Densely sampled radio light curves at 1.3 and $2 \mathrm{~cm}$ observed with the VLA (R. M. Herrnstein et al. 2003, in preparation).

in both the visibility and imaging domains. In the visibility domain, we measured the amplitude of the baselines of $\sim 20 \mathrm{k} \lambda$ and longer. The flux density was double-checked by constructing images with the self-calibrated (phase-only) visibility data. The measurements of the point-source flux density were done by deconvolving the telescope beam and taking out the contribution from surrounding components using IMFIT in AIPS. The flux densities derived from the two domains are in an agreement within $10 \%$ of the mean value. This additional $10 \%$ level of the uncertainty in the measurements is mainly due to the confusion from the surrounding emission and is added to the final error assessment. The error bars of Sgr A* are derived from the quadrature addition of the uncertainty in flux density calibration and the uncertainty due to the confusion. The typical uncertainty at $1 \mathrm{~mm}$ is in the range of $10 \%-20 \%$.

\section{RESULTS}

\subsection{Light Curve and Flares}

Figure 2 shows the SMA light curve at $1.3 \mathrm{~mm}$, suggesting that Sgr A* varies significantly. A few "flares" were observed from Sgr A*, while the calibrators show secular variations with opposite drifts in flux density over the past year (Fig. 2a). Three flares were observed over a $1 \mathrm{yr}$ period. Both the $2001 \mathrm{March}$ and 2002 February flares (flare 1 and flare 3 as marked in Fig. $2 b$ ) were partially observed in their decreasing phase. The 2001 July flare (flare 2) was observed covering an entire cycle from its inception to a slow decrease back to its steady state value. Flare 1, which started at $4.1 \pm 0.5 \mathrm{Jy}$ after an unobserved peak and decreased to $1.1 \pm 0.15 \mathrm{Jy}$ within less than 3 months, appeared to be relatively stronger than others. The rising time for flare 2 was about 2-3 weeks, reaching a peak of $3.2 \pm$ $0.3 \mathrm{Jy}$ on 2001 July 10. Then, a slow decrease lasted about 
40-50 days. We were not able to observe Sgr A* for the next 3 months owing to the proximity to the Sun. The monitoring program was resumed in 2002 February. A tail of a possible flare (flare 3) was observed in early 2002.

\subsection{Day-to-Day Variability and Nondetection Limit on Intraday Variability}

Based on the sparse data, marginal day-to-day variations at a level of 2-3 $\sigma$ (or 20\%-30\%) were observed during flare 1 and flare 2 as well as in later May 2002.

Intraday variations on short timescales were searched based on the 24 epochs of observations at $1.3 \mathrm{~mm}$. We averaged the data in a 5 minute bin and checked the visibility plots for each baseline. We also averaged all baselines together and checked intraday light curves. No evidence for significant variations on a timescale of $\sim 1 \mathrm{hr}$ has been found, i.e., a variability quantity $\left(S_{\max }-S_{\min }\right) /\left(S_{\max }+S_{\min }\right)<20 \%$, where $S_{\max }$ and $S_{\min }$ are the maximum and minimum flux densities at $1.3 \mathrm{~mm}$, respectively, in a single observing track of $6 \mathrm{hr}$ or less.

We also searched for periodic signals in a period ranging from 10 minutes to $2.5 \mathrm{hr}$ based on the observations of $5.5 \mathrm{hr}$ on 2002 May 29. There is a possible oscillation signal with a frequency of $1.1 \times 10^{-4} \mathrm{~Hz}$ (or $2.5 \mathrm{hr}$ in period) on an east-west baseline. This oscillation signal can be well modeled as a structure due to interference between Sgr A* and surrounding components. Combining all baselines, a $3 \sigma$ nondetection limit of a periodic signal from $\mathrm{Sgr} \mathrm{A}^{*}$ at a level of $\sim 5 \%$ can be inferred.

\subsection{Spectrum during a Flare}

We also observed Sgr A* at $0.87 \mathrm{~mm}$ with the SMA on 2001 March 22. We observed $\operatorname{Sgr} A^{*}\left(S_{0.87 \mathrm{~mm}}=6.7 \pm 1.5 \mathrm{Jy}\right)$ at the submillimeter band using the three-element array of the partially completed SMA. Figure $3 a$ shows a spectrum derived from the mean flux density determined from the multiple observations within 2 weeks of the peak of flare 1 . The error bars were derived from the quadrature addition of the standard deviation and the maximum error in individual measurements. The spectral index $\alpha\left(S_{\nu} \propto \nu^{\alpha}\right)$ appears to be $0.1 \pm 0.1$ at $100 \mathrm{GHz}$ and below and $1.5_{-1.1}^{+1.0}$ between 232 and $345 \mathrm{GHz}$, suggesting a break frequency in spectral index of $\sim 100 \mathrm{GHz}$ or higher. A flux density excess toward submillimeter wavelengths has been observed (Zylka, Mezger, \& Lesch 1992; Serabyn et al. 1997; Falcke et al. 1998).

The overall spectrum can fit two power-law components, i.e., $S_{\nu}=S_{1}\left(\nu / \nu_{1}\right)^{\alpha_{1}}+S_{2}\left(\nu / \nu_{2}\right)^{\alpha_{2}}$. Three sets of a combination of $\alpha_{1}$ and $\alpha_{2}$ are used in the fitting. First, for $\alpha_{1}=0.0$ and $\alpha_{2}=2$ (Fig. 3, dashed lines), the submillimeter component corresponds to the thermal synchrotron emission either arising from the inner region of the accretion disk (e.g., Liu \& Melia 2002) or produced from a jet nozzle (Falcke \& Markoff 2000). Second, for $\alpha_{1}=$ 0.1 and $\alpha_{2}=2.5$ (Fig. 3, solid lines), the spectral index of 2.5 suggests that a homogeneous opaque, nonthermal synchrotron source might be present in the inner region of the accretion flow. Such a model appears to be plausible if one considers the nonthermal synchrotron particles to be accelerated inside the compact source, perhaps within a jet nozzle, as has been proposed for the case of NGC 4258 (Yuan et al. 2002b). Finally, if the low-frequency component has an exponential cutoff, i.e., $\sim S_{1}\left(\nu / \nu_{1}\right)^{0.25} \exp \left(-\nu / \nu_{0}\right)$, at $\nu_{0} \sim 75 \mathrm{GHz}$, a smaller value of $\alpha_{2} \sim 1.5$ (Fig. 3, dash-dotted lines) for the submillimeter component is also consistent with a spectrum produced from the ADAF in which a gradient of $T_{e}$ depresses the rising part of the spectrum (Narayan et al. 1998). The observed spectrum suggests an opaque nature of the submillimeter component at 1.3 and
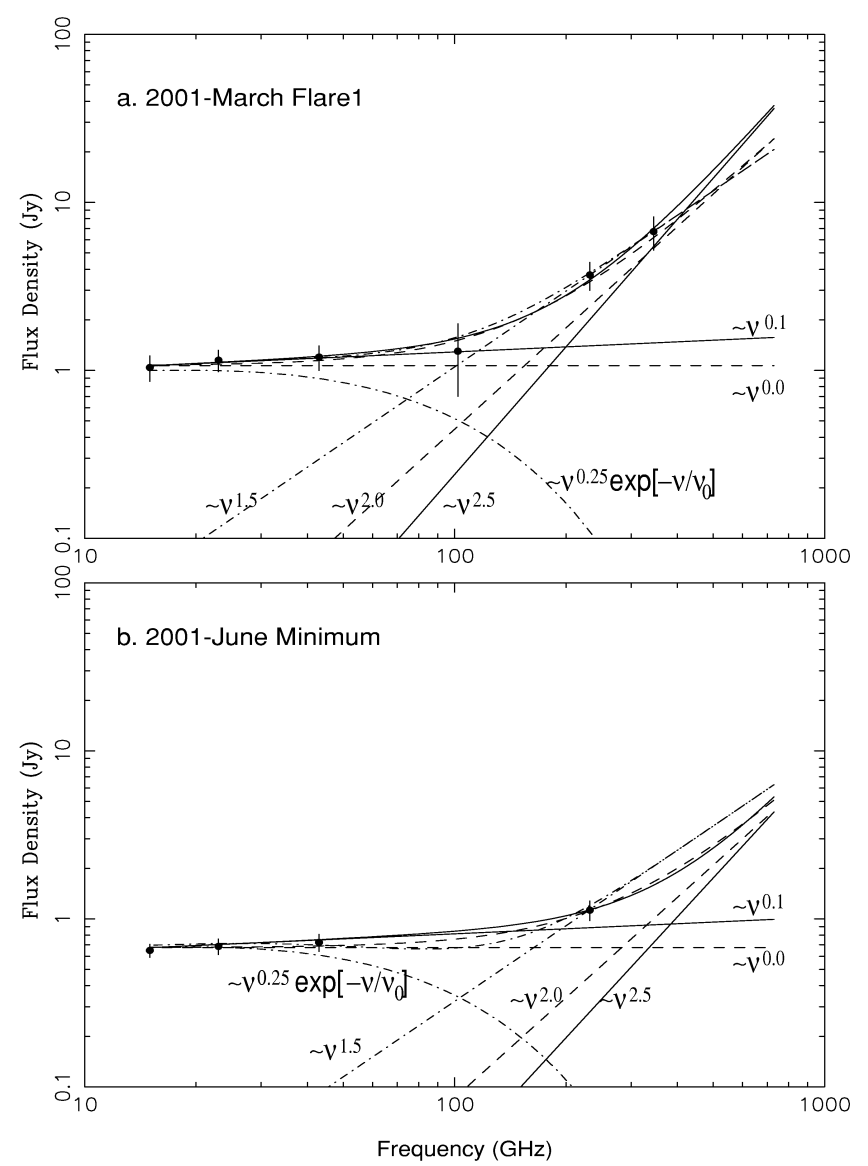

FIG. 3.-(a) Mean spectrum of Sgr A* made from the observations near the peak of flare 1 . The flux densities of $6.7 \pm 1.5$ and $3.7 \pm 0.7 \mathrm{Jy}$ at 0.87 and $1.3 \mathrm{~mm}$ are derived from the SMA observations. The flux density of $1.3 \pm 0.6 \mathrm{Jy}$ at $3 \mathrm{~mm}$ is averaged from the measurements made with the Nobeyama Millimeter Array (Tsutsumi, Miyazaki, \& Tsuboi 2002). The data $(1.2 \pm 0.2,1.15 \pm 0.17$, and $1.04 \pm 0.18 \mathrm{Jy})$ at $0.7,1.3$, and $2 \mathrm{~cm}$ are derived from the VLA monitoring observations (R. M. Herrnstein et al. 2003, in preparation). The curves represent a resultant spectrum of two power-law components (straight lines) for various sets of spectral indices corresponding to different emission processes for the submillimeter component (see $\$ 3.3$ ). (b) Spectrum of Sgr A* made from the observations on the same date (2001 June 17) during a minimum of the Sgr A* light curves (see Fig. 2). The flux density of $1.1 \pm 0.15$ at $1.3 \mathrm{~mm}$ is measured with the SMA. The rest of the measurements $(0.72 \pm 0.09,0.69 \pm 0.07$, and $0.65 \pm 0.06 \mathrm{Jy}$ at $0.7,1.3$, and $2 \mathrm{~cm}$ ) are made from the VLA observations.

perhaps $0.87 \mathrm{~mm}$. Observations at the shorter submillimeter wavelengths appear to be critical to differentiate between the models. The spectrum in a minimum state is also shown (Fig. $3 b$ ). The excess at $1.3 \mathrm{~mm}$ appears to be less significant.

\subsection{Correlation with the VLA Data}

The SMA data at $1 \mathrm{~mm}$ appear to show a correlation with the light curves observed with the VLA. The SMA light curve shows three flares from Sgr A* in the past year from 2001 March to 2002 May. The variation in flux density that we observed in the SMA light curve suggests that flares are constantly occurring in this source.

A quantitative analysis of cross-correlation properties between the light curves at $1.3 \mathrm{~mm}$ and $1.3 \mathrm{~cm}$ has been carried out. Owing to the sparse data sampling, large uncertainty remains in the cross-correlation analysis for individual flares (J.-H. Zhao et al. 2003, in preparation). However, a global delay between the $1.3 \mathrm{~mm}$ and $1.3 \mathrm{~cm}$ light curves can be searched for using the 


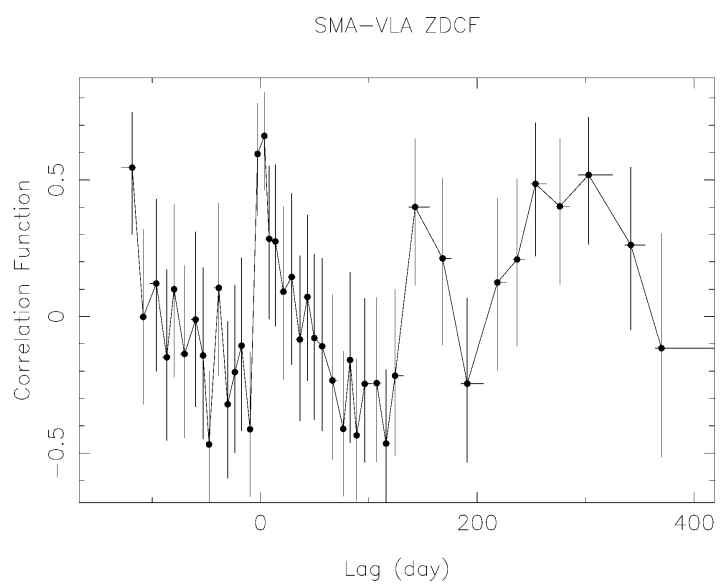

FIG. 4.-ZDCF of the SMA data at $1.3 \mathrm{~mm}$ with the VLA data at $1.3 \mathrm{~cm}$

$z$-transformed discrete correlation function (ZDCF) without interpolating in the temporal domain (Alexander 1997). With no prior models assumed, the ZDCF is a reliable and efficient method to search for a delay. Figure 4 shows the ZDCF between the SMA and VLA light curves at $1.3 \mathrm{~mm}$ and $1.3 \mathrm{~cm}$. The peak in the correlation function corresponds to a delay of $4_{-1}^{+2}$ days. A noticeable asymmetric shape of the ZDCF near the zero lag shows a significant excess in cross-correlated power toward the positive lags, suggesting that the true global delay is $t_{\text {delay }}>3$ days. The ZDCF indicates that flares at $1.3 \mathrm{~mm}$ start first.

In addition, the strong X-ray flare with a timescale of $\sim 1 \mathrm{hr}$, observed by Baganoff et al. (2001), occurred about 10 days earlier than a radio peak observed in all three VLA monitoring bands. During flare 2 (2001 July), Chandra observed Sgr A* on 2001 July 14, a few days past the $1 \mathrm{~mm}$ peak, but no X-ray flares were observed (F. K. Baganoff 2001, private communication).

\section{DISCUSSION AND SUMMARY}

The SMA observations have shown that Sgr A* varies significantly at $1 \mathrm{~mm}$ during the course of SMA monitoring from 2001 March to 2002 May. The derived lags from a crosscorrelation analysis appear to be good evidence that the flaring occurs from the inside out starting from short wavelengths and then continuing to longer wavelengths. In the jet-ADAF model, connecting a jet outflow with the ADAF (Yuan et al. 2002a), the submillimeter excess is thought to arise from a sum of the emission from both the ADAF and the jet nozzle.

On the other hand, from the observations with the SMA and the VLA, further constraints on the models can be derived. Taking a global delay time of $t_{\text {delay }}>3$ days and the source size of $40 R_{\mathrm{Sc}}$, an expansion velocity $v_{\exp } \sim 1200 \mathrm{~km} \mathrm{~s}^{-1}$, or less than $0.004 c$, is inferred. The expansion velocity appears to be far below the escape velocity of $0.1 c$ at $r \sim 40 R_{\mathrm{Sc}}$. The bulk kinetic energy associated with the flares appeared to be too small to power a noticeable collimated jet in $\mathrm{Sgr} \mathrm{A}^{*}$. In addition, the break in the spectral index at $\sim 3 \mathrm{~mm}$ also indicates that a large fraction of the flaring plasma might well be confined within the characteristic radius at $3 \mathrm{~mm}$. However, the possible outflow tries to expand to a larger scale. The data presented here do not exclude the possibility of a strong flare that can lead to an observable jetlike structure. The inferred small expansion velocity may imply that other processes contribute to the transport of high-energy particles; e.g., diffusion and convection may also play a role in powering $\mathrm{Sgr} \mathrm{A} *$ at lower radio frequencies.

The timescale (weeks) of $1 \mathrm{~mm}$ flares differs from the timescale (1 hr) of the X-ray flare (Baganoff et al. 2001). The lack of strong flares on a short timescale at $1 \mathrm{~mm}$ places a critical constraint on the models of the inverse Compton scattering as has been proposed for the short-duration X-ray flares (Falcke \& Markoff 2000; Markoff et al. 2001; Liu \& Melia 2002). Considering the opaque nature of the submillimeter component at $1.3 \mathrm{~mm}$, the X-ray flares could hide at $1.3 \mathrm{~mm}$ owing to selfabsorption. Alternatively, the flares at submillimeter wavelengths might be a result of collective mass ejections associated with the $\mathrm{X}$-ray flares that originate from the inner region of the accretion flows near the event horizon of the supermassive black hole at the Galactic center.

We would like to thank the SMA staff from both the Smithsonian Astrophysical Observatory and the ASIAA for supporting this monitoring program. We are grateful to Jim Moran and Heino Falcke (the referee) for their valuable comments. J.-H. Z. thanks Irwin Shapiro for his initial suggestion of the SMA monitoring program. The VLA is operated by the National Radio Astronomy Observatory (NRAO). The NRAO is a facility of the National Science Foundation operated under cooperative agreement by Associated Universities, Inc.

\section{REFERENCES}

Alexander, T. 1997, in Astronomical Time Series, ed. D. Maoz, A. Sternberg, \& E. Leibowitz (Dordrecht: Kluwer), 163

Baganoff, F. K., et al. 2001, Nature, 413, 45

Bower, G. C., McGary, R. S., Zhao, J.-H., \& Goss, W. M. 2002a, AAS Meeting, 199, 85.08

Bower, G. C., et al. 2002b, ApJ, submitted

Brown, R. L., \& Lo, K. Y. 1982, ApJ, 253, 108

Doeleman, S., et al. 2001, AJ, 121, 2610

Eckart, A., Genzel, R., Ott, T., \& Schödel, R. 2002, MNRAS, 331, 917

Falcke, H. 1999, in ASP Conf. Ser. 186, The Central Parsecs of the Galaxy, ed. H. Falcke, A. Cotera, W. J. Duschl, F. Melia, \& M. J. Rieke (San Francisco: ASP), 113

Falcke, H., Mannheim, K., \& Biermann, P. L. 1993, A\&A, 278, L1

Falcke, H., \& Markoff, S. 2000, A\&A, 362, 113

Falcke, H., et al. 1998, ApJ, 499, 731

Ghez, A., Morris, M., Becklin, E., Tanner, A., \& Kremenek, T. 2000, Nature, 407, 349

Liu, S., \& Melia, F. 2002, ApJ, 566, L77

Markoff, S., Falcke, H., Yuan, F., \& Biermann, P. L. 2001, A\&A, 379, L13

Moran, J. M. 1998, Proc. SPIE, 3357, 208

Narayan, R., Mahadevan, R., Grindly, J., Popham, R., \& Cammie, C. 1998, ApJ, 492, 554
Serabyn, E., Carlstrom, J., Lay. O., Lis, D., Hunter, T., \& Lacy, J. 1997, ApJ, 490, L77

Tsuboi, M., Miyazaki, A., \& Tsutsumi, T. 1999, in ASP Conf. Ser. 186, The Central Parsecs of the Galaxy, ed. H. Falcke, A. Cotera, W. J. Duschl, F. Melia, \& M. J. Rieke (San Francisco: ASP), 105

Tsutsumi, T., Miyazaki, A., \& Tsuboi, M. 2002, AAS Meeting, 200, 44.09

Wright, M., \& Backer, D. C. 1993, ApJ, 417, 560

Yuan, F., Markoff, S., \& Falcke, H. 2002a, A\&A, 383, 854

Yuan, F., Markoff, S., Falcke, H., \& Biermann, P. L. 2002b, A\&A, 391, 139

Zhao, J.-H. 2002, Newsl. Galactic Cent. Res., 15, 4

Zhao, J.-H., Bower, G. C., \& Goss, W. M. 2001, ApJ, 547, L29

Zhao, J.-H., Ekers, R. D., Goss, W. M., Lo, K. Y., \& Narayan R. 1989, in IAU Symp. 136, The Center of the Galaxy, ed. M. Morris (Dordrecht: Kluwer), 535

Zhao, J.-H., \& Goss, W. M. 1993, in Sub-Arcsecond Radio Astronomy, ed. R. J. Davis \& R. S. Booth (Cambridge: Cambridge Univ. Press), 38

Zhao, J.-H., Goss, W. M., Lo, K. Y., \& Ekers, R. D. 1992, in ASP Conf. Ser. 31, Relationships between Active Galactic Nuclei and Starburst Galaxies, ed. A. V. Filippenko (San Francisco: ASP), 295

Zylka, R., Mezger, P. G., \& Lesch, H. 1992, A\&A, 261, 119 